\title{
Microsurgical Anatomy of the Orbit: The Rule of Seven
}

\author{
Carolina Martins, ${ }^{1,2}$ Isabel Eugênia Costa e Silva, ${ }^{3}$ Alvaro Campero, ${ }^{2}$ Alexandre Yasuda, ${ }^{2}$ \\ Luiz Roberto Aguiar, ${ }^{4}$ Marcos Tatagiba, ${ }^{5}$ and Albert Rhoton Jr. ${ }^{2}$
}

${ }^{1}$ Medical School of Pernambuco, IMIP, 1393 Recife, PE, Brazil

${ }^{2}$ Department of Neurosurgery, University of Florida, Gainesville, FL 32611, USA

${ }^{3}$ Department of Neurosurgery, Hospital da Restauração, Recife, PE, Brazil

${ }^{4}$ Medical School, Pontifical Catholic University of Paraná, 80215 Curitiba, PR, Brazil

${ }^{5}$ Department of Neurosurgery, Eberhard University of Tübingen, 72074 Tübingen, Germany

Correspondence should be addressed to Alvaro Campero, alvarocampero@yahoo.com.ar

Received 31 May 2010; Accepted 5 August 2010

Academic Editor: Robert J. Spinner

Copyright (C) 2011 Carolina Martins et al. This is an open access article distributed under the Creative Commons Attribution License, which permits unrestricted use, distribution, and reproduction in any medium, provided the original work is properly cited.

\begin{abstract}
The orbits are paired structures, located on the anterior part of the face. Morphologically, each orbit is a four sided pyramid with a posterior apex and anterior base. In the orbit, all openings are arranged around the base, apex or between the orbital walls. An anatomical characteristic of the orbit is that structures are arranged in groups of seven: there are seven bones, seven intraorbital muscles and seven nerves in the orbit. Tumors confined within the periorbita in the anterior two thirds of the orbit can often be approached extracranially, but those located in the apical area, and especially those on the medial side of the optic nerve, often require a transcranial approach. Thus, knowledge of orbital osteology is paramount in adequately choosing and performing an orbital approach. Understanding the critical topographical elements in this area helps to classify an orbital lesion and provides for a solid basis in choosing the most adequate intraorbital route for its treatment.
\end{abstract}

\section{Introduction}

The orbits are paired structures, located on the anterior part of the face and protected by the lids (Figures 1(a) and 1(b)). Each orbit can be compared to a tiny jewel box that has very precious contents, all carefully wrapped in fat tissue. They can also be compared to a main room, to which access is gathered through a prechamber, the cavernous sinus.

Morphologically, each orbit is a four-sided pyramid with a posterior apex, anterior base and a medially tilted axis (Figures $1(\mathrm{c})$ and $1(\mathrm{~d})$ ). Although simple, this fact constitutes the basis of the human stereoscopic vision and allows for understanding the location of orbital foramina.

In the orbit, all openings are arranged around the base, apex or between the orbital walls (Figure $1(\mathrm{~d})$ ). Along the base are the infraorbital and supraorbital canals and the zygomaticofacial foramina; between the roof and the lateral wall are the superior orbital fissure and the lacrimal foramen; between the roof and medial wall are the optic, anterior, and posterior ethmoidal canals; between the lateral wall and floor is the inferior orbital fissure, and between the medial wall and orbital floor is the cranial opening of the nasolacrimal duct.

Another anatomical characteristic of the orbit is that structures are arranged in groups of seven: there are seven bones, seven intraorbital muscles, and seven nerves in the orbit.

\section{Orbital Bones}

Seven are the bones forming the orbit: frontal, ethmoid, lacrimal, sphenoid, zygomatic, palatine, and maxilla (Figures 1 (e) and 2). In three out of the four orbital walls, these bones are arranged in pairs, with exception of the medial wall.

The largest component of the medial wall is the ethmoid (Figure 2(a)). The quadrangular orbital plate of the ethmoid constitutes the center of the medial orbital wall and separates the orbit from the medially located nasal cavity. The orbital plate of the ethmoid articulates superiorly with the medial 


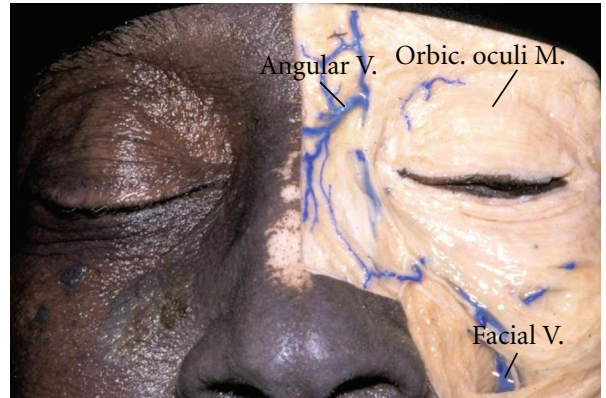

(a)

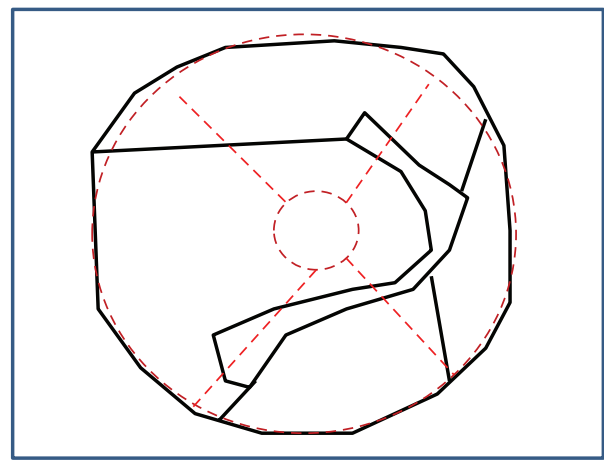

(c)

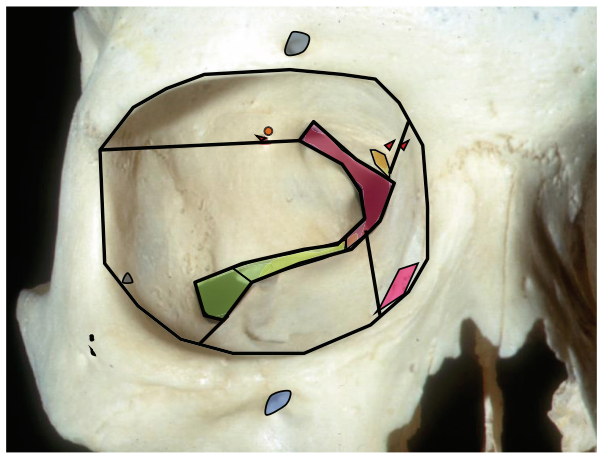

(e)

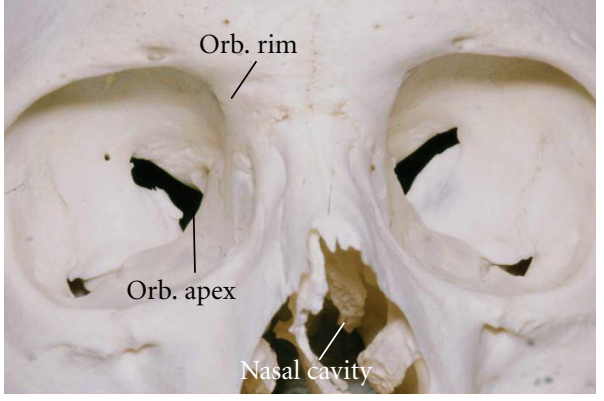

(b)

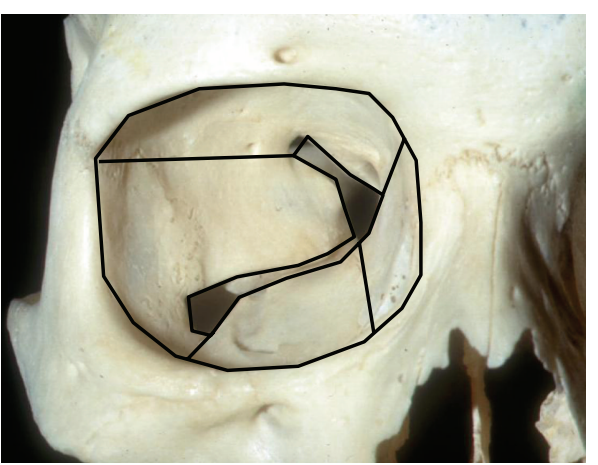

(d)

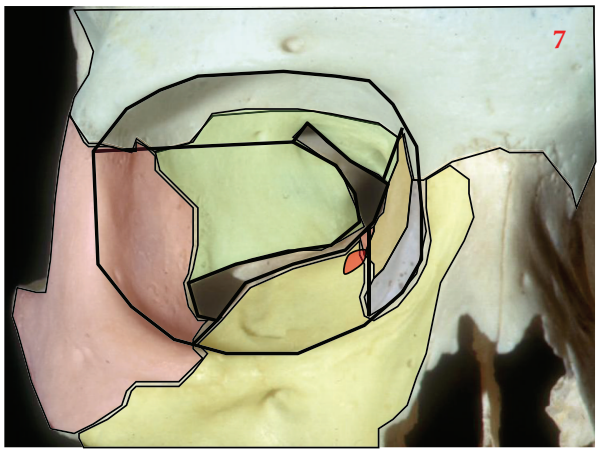

(f)

FIgURE 1: (a) The orbits are paired structures, located on the anterior part of the face and protected by the lids. The skin and subcutaneous tissue have been removed on the left, to expose the orbicularis oculi muscle. The orbicularis oculi surrounds the orbital rim, extends into the lids, and encircle the upper part of the nasolacrimal duct. (b)-(d) Understanding the bony orbit is the first step in successfully choosing and performing an orbital approach. Seven bones form each orbit, and they are arranged to form a four-sided pyramid with a posterior apex and anterior base as shown in red dots (c). This pyramid, however, is not straight, but displays a laterally tilted axis (black outline in (c) and (d)). Although simple, this fact constitutes the basis of the human stereoscopic vision and allows for understanding the location of orbital foramina. (e) In the orbit, all openings are arranged around the base, apex or between the orbital walls. Along the base are the infraorbital (light blue) and supraorbital (dark blue) canals, the zygomatico-orbital foramen (gray), and the zygomaticofacial foramina (black); between the roof and the lateral wall are the superior orbital fissure (purple) and the lacrimal foramen (orange); between the roof and medial wall are the optic (yellow), anterior, and posterior ethmoidal (red) canals; between the lateral wall and floor is the inferior orbital fissure (green), and between the medial wall and the floor is the cranial opening of the nasolacrimal duct (pink). (f) The seven bones forming the orbit have been highlighted in color: frontal (blue), ethmoid (brown), lacrimal (grey), sphenoid (green), zygomatic (pink), palatine (red), and maxilla (yellow). In three of the four orbital walls, these bones are arranged in pairs, with exception of the medial wall.

edge of the orbital plate of the frontal bone (Figure 2(c)). The anterior and posterior ethmoidal notches that exist in both of these plates, when combined, form the anterior and posterior ethmoidal canals (Figure 2(d)). These canals transmit the ethmoidal branches of the nasociliary nerve of the ophthalmic division of the trigeminal nerve and the branches of the ophthalmic artery that pass further to supply the sinus mucosa and the dura mater of the frontal pole and falx.

The cranial openings of the ethmoidal canals are related with the anterior and posterior limits of the ethmoidal cribriform plate and help dividing the nasal cavity roof 
and anterior fossa floor into frontal, cribriform, and planum areas. The ethmoidal canals also help dividing the orbital area into bulbar, retrobulbar, and apical parts. This anatomical fact is useful during intracranial exploration of the anterior fossa [1] and endonasal approaches to the anterior fossa and orbit.

Inferiorly, the ethmoid articulates with the orbital plate of the maxilla (Figure 2(d)). At the most anterior portion of the orbit, the presence of the frontal process of the maxilla, forming most of the medial rim of the orbit, determines the presence of a gap, which is filled in by the upper portion of the lacrimal bone (Figures 2(e) and 2(f)). The upper part of the lacrimal bone is therefore located between the anterior edge of the ethmoid and the frontal process of the maxilla. The lacrimal is a curved plate of bone, which forms the anteromedial $2 / 3$ of the cranial opening of the nasolacrimal duct (Figure 2(g)).

Posteriorly, the ethmoid articulates with the body of the sphenoid, completing the medial wall of the orbit (Figure 2(h)).

In fact, the sphenoid contributes to the bony formation of the orbit through its body, lesser and greater wings. The lesser wings combine with the orbital plates of the frontal bone to form the orbital roof (Figure 2(i)), and the greater wings-laterally directed-join the orbital surface of the zygomatic bone to form the lateral orbital wall (Figures 2(j)2(1)).

Understanding the bony formation of the lateral wall of the orbit is important from the surgical standpoint (Figures $2(\mathrm{~m})$ and $2(\mathrm{n}))$. The greater wing of the sphenoid faces the orbit, along the exocranial side, and is the anterior limit of the middle fossa, on the endocranial surface. The zygomatic bone, in contrast, has no cerebral surface. The zygoma faces the orbit and-through its opposite surfaceforms the anterior limit of the temporal fossa, where the temporal muscle is located. This anatomical fact is the basis of the lateral orbital approaches, in which by displacing the temporal bone and performing a pure zygomatic osteotomy, orbital lesions can be reached, without the need of a combined, cranio-orbital approach.

The combination of the greater sphenoid wing and the zygomatic bone also forms the posterior lip of the inferior orbital fissure (Figure 2(o)). The anterior lip of this fissure is formed mostly by the orbital plate of the maxilla and the posteromedial part is formed by the orbital process of the palatine bone (Figure 2(p)).

The palatine bones (Figure 2Q) have a horizontal part, which form the posterior part of the hard palate and a vertical part. The vertical part has a posteromedial process, directed to the sphenoid body - the sphenoid process - and an orbital process, directed anterolaterally, consisting of a single air cell and abutting along the floor of the orbit, between the maxilla and the ethmoid. The palatine bone pairs thus with the maxilla to form the orbital floor. They face posteriorly, across the pterygomaxillary fissure, the pterygoid processes of the sphenoid bone (Figures 2(r) and 2(s)).

The inferior orbital fissure is an important surgical landmark in the orbit. Through the most medial part of this fissure, the orbit communicates with the pterygopalatine fossa and, through this, with the nasal cavity (Figures 2(t) and $2(\mathrm{u}))$. Laterally the fissure brings the orbit in contact with the temporal and infratemporal fossae. The lateral part of the fissure is filled only with smooth muscle and adipose tissue, making it a suitable point to bear the bony cuts needed to remove part of the roof and lateral walls with corresponding orbital rim, as in the cranio-orbito-zygomatic approaches (Figure 2(u)).

Beyond the optic canal and superior orbital fissure, the bony orbit is covered by an extension of the cranial dura mater, the periorbit (Figure 3(a)). The periorbit or orbital dura envelops and holds the intraorbital structuresespecially the orbital fat. This structure also protects the orbital contents from tumoral invasion and inflammation.

The presence of the periorbit allows classifying the orbital lesions in intradural-when deep to the periorbit-and extradural-when located between the periorbit and the bony orbit.

\section{Orbital Muscles and Vessels}

With the exception of the sphincter oculi muscle, located along the base of the orbital pyramid, seven are the intraorbital muscles (Figure 3(b)). Of these, only the inferior oblique muscle is attached to the medial orbital wall. The four recti and the superior oblique muscles attach along the orbital apex in and around a common annular tendon or fibrous ring, the annulus of Zinn (Figure 3(c)). The superior oblique muscle passes through the trochlea, a round tendon attached to the trochlear fossa of the frontal bone on the superomedial part of the orbit. Lesion to the trochlea during fractures of medial orbital wall may result in downward gaze dysfunction and diplopia.

The annulus inserts along the orbital apex, encircling the orbital opening of the optic canal and the central part of the superior orbital fissure. Structures coursing through the annulus are the optic nerve and ophthalmic artery, the oculomotor and abducens nerves, and the ophthalmic division of the trigeminal nerve. The trochlear nerve and the frontal and lacrimal divisions of the trigeminal nerve course outside the annulus.

The ophthalmic artery is the major supply to the orbit (Figure 4(a)). It is a branch of the supraclinoid portion of the internal carotid artery, in most cases. This vessel follows the optic nerve in the optic canal and orbit and is the responsible for the supply of orbital structures, including optic and lacrimal apparatus, muscles and nerves. The origin of the ophthalmic artery is usually medial to the anterior clinoid process, below the optic nerve (Figure 4(b)). At the optic canal the artery has already passed lateral to nerve (Figure 4(c)). This anatomical fact must be remembered during opening of the falciform ligament after removal of the anterior clinoid, to prevent iatrogenic lesion to this artery. Approximately $8 \%$ of ophthalmic arteries arise in the cavernous sinus rather than in the subarachnoid space [2]. The ophthalmic artery may also arise as duplicate arteries of nearly equal size [3]. It may also infrequently arise as a branch of the middle meningeal artery [4]. 


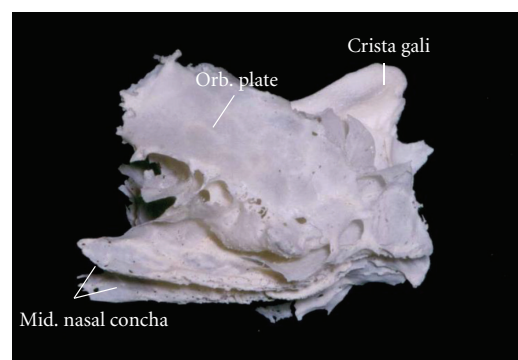

(a)

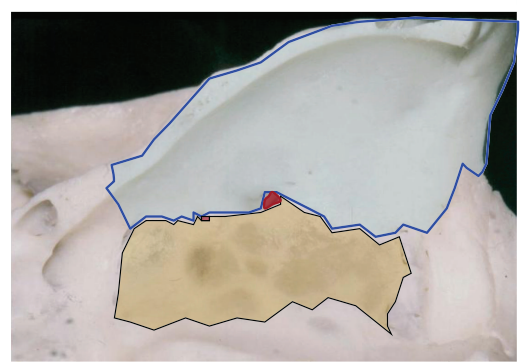

(c)

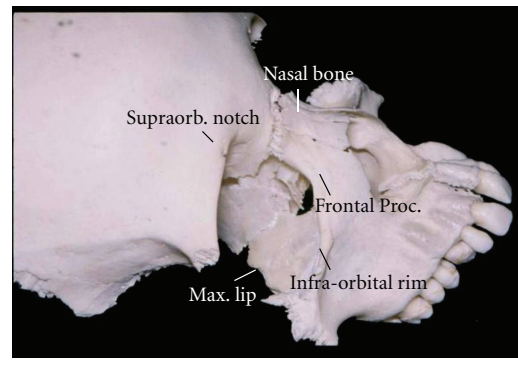

(e)

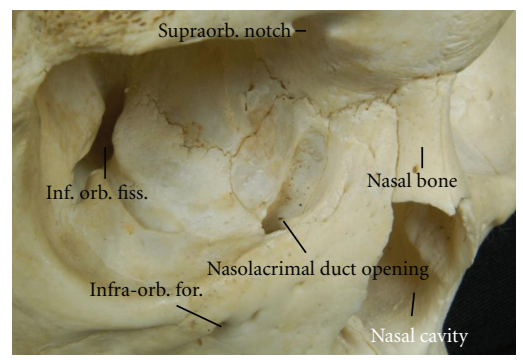

(g)

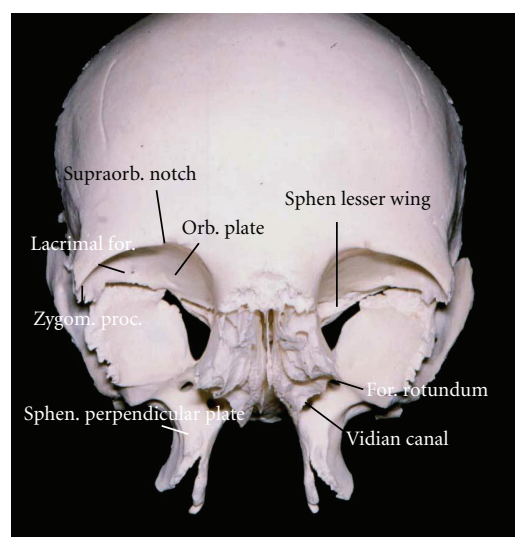

(i)

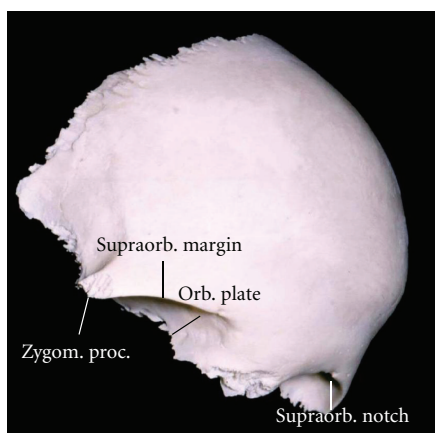

(b)

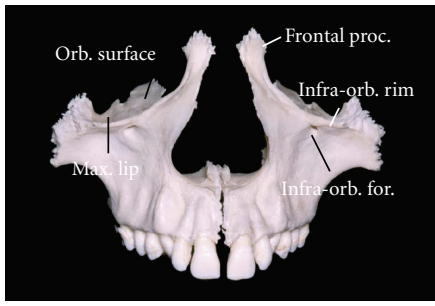

(d)

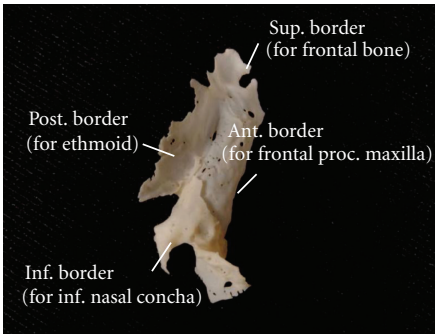

(f)

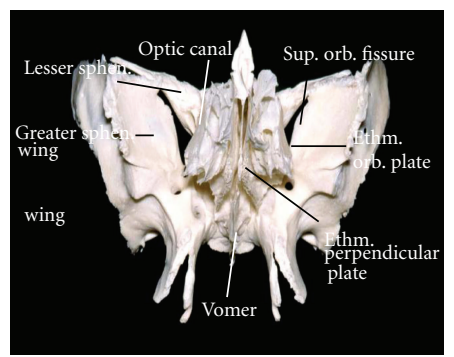

(h)

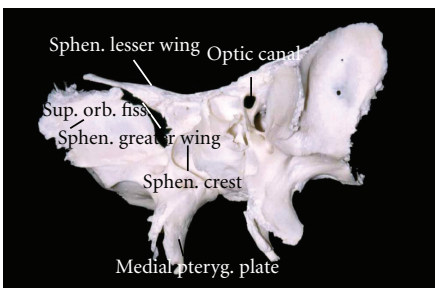

(j)

FIgURE 2: Continued. 


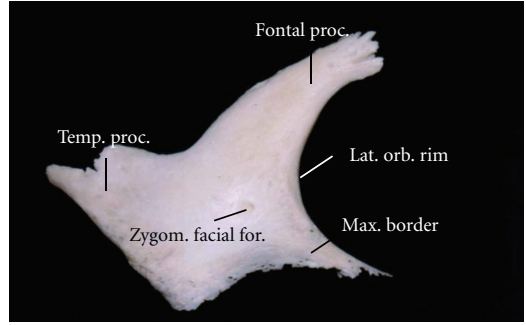

(k)

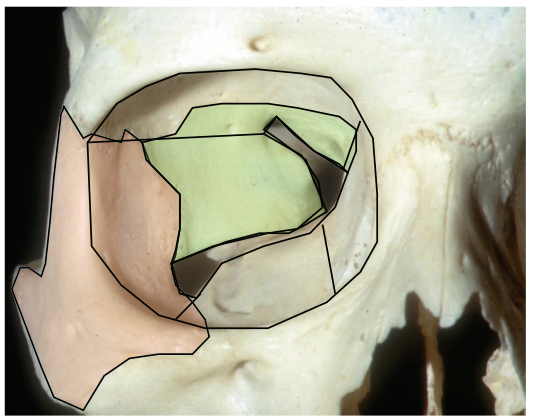

(m)
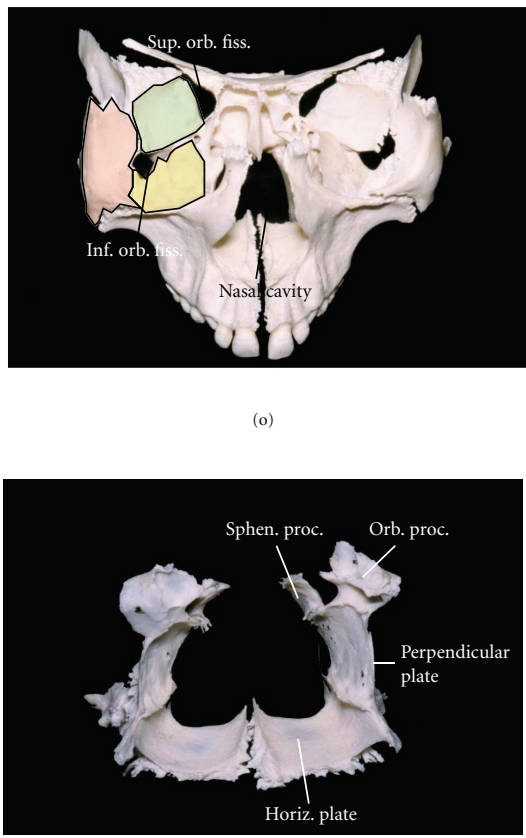

(q)

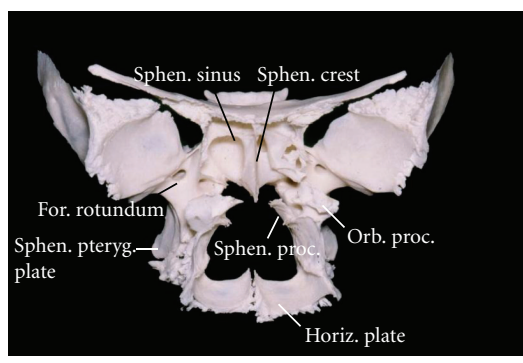

(r)
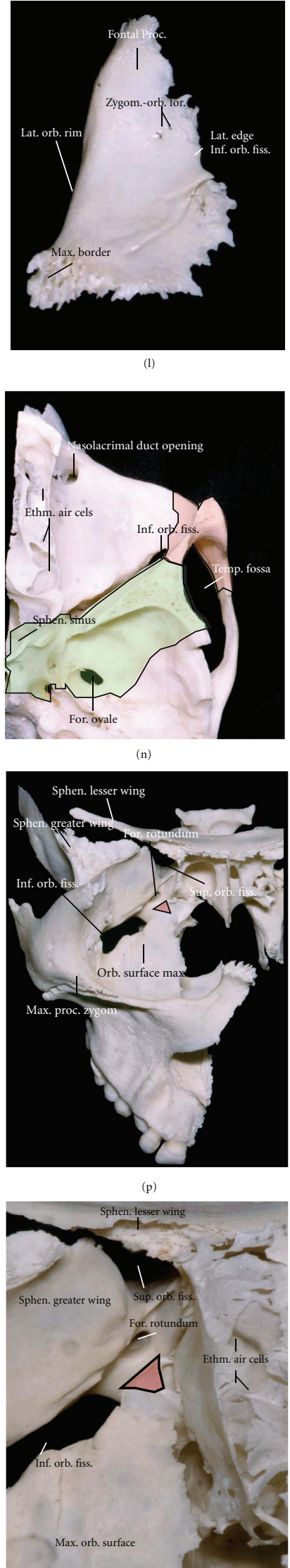

(s)

FIgUre 2: Continued. 

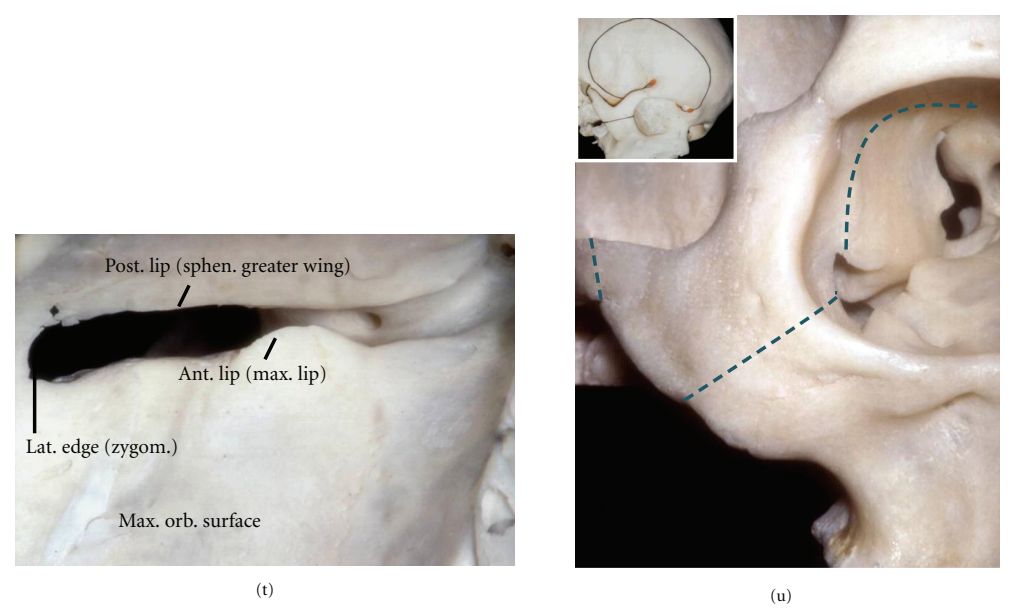

FIGURE 2: kull bones are added to gradually rebuild the bony orbit, starting along the medial orbital wall. (a) The ethmoid. The quadrangular orbital plate of the ethmoid constitutes the center of the medial orbital wall and separates the orbit from the medially located nasal cavity. (b) The frontal bone. The orbital plate of the ethmoid articulates superiorly with the medial edge of the orbital plate of the frontal bone. The anterior and posterior ethmoidal notches that exist in both of these plates, when combined, form the anterior and posterior ethmoidal canals. (c) Parasagittal cut through the right orbit, showing the combination of the orbital plates of the frontal (blue) and ethmoidal bone (brown) to form the ethmoidal canals (red). (d) Maxillae. Inferiorly, the ethmoid articulates with the orbital surface of the maxilla. (e) Ethmoid, frontal bone, and maxilla are combined. (f) Lacrimal bone. The upper part of the lacrimal bone is a thin plate of bone located between the anterior edge of the ethmoid and the frontal process of the maxilla. (g) In the articulated skull, the lacrimal bone can be recognized as a curved plate of bone, which forms the anteromedial $2 / 3$ of cranial opening of the nasolacrimal duct. (h) Posteriorly, the ethmoid articulates with the body of the sphenoid, therefore completing the medial wall of the orbit. (i) The sphenoid contributes to the bony formation of the orbit through its body, lesser and greater wings. (j) The greater sphenoid wings, laterally directed, join the orbital surface of the zygomatic bone to form the lateral orbital wall. (k) The zygomatic bone. Lateral surface. (l) The zygomatic bone. Orbital surface. (m) The lateral wall of the orbit is formed by the combination of the orbital surfaces of the greater sphenoid wing (green) and the zygomatic bone (pink). (n) An axial cut has been made through the right orbit, removing its roof. The greater wing of the sphenoid (green) is, on the endocranial side, the anterior limit of the middle fossa. The zygomatic bone (pink), in contrast, forms the anterior limit of the temporal fossa, where the temporal muscle is located. (o) The sphenoid (green), maxillae (yellow) and zygomatic bones (pink) have been combined. (p) The anterior lip of the inferior orbital fissure is formed by the orbital surface of the maxilla and the orbital process of the palatine bone (red). (q) The palatine bones have a horizontal part, which form the posterior part of the hard palate, and a vertical part (perpendicular plate). (r)-(s). The palatine bones face posteriorly the pterygoid processes of the sphenoid bone, across the pterygomaxillary fissures while the orbital process (red) abuts along the floor of the orbit, between the maxilla and the ethmoid. (t) The inferior orbital fissure is located along the lateral wall and the floor of the orbit. (u) The inferior orbital fissure is an important surgical landmark in the orbit. The lateral part of the fissure is filled with smooth muscle and fat tissue, making it a suitable point to bear the bony cuts needed to remove part of the roof and lateral walls with corresponding orbital rim, as in the cranio-orbito-zygomatic approaches (Insert).

Once inside the orbit, the ophthalmic artery passes, in most cases, above the optic nerve (Figure 4(d)). At this crossing point it usually gives rise to the central retinal artery (Figure $4(\mathrm{e})$ ). Working along the lateral orbital space, it is vital to avoid blind coagulation, once inadvertent coagulation of the retinal artery results in loss of vision.

The ophthalmic artery gives rise to the central retinal, supraorbital, medial palpebral, lacrimal, long and short ciliary, infratroclear, supratroclear, and dorsal nasal arteries. The ophthalmic artery also sends dural branches and may thus become involved in the supply of dural-based lesions [1]. In this role, two sets of branches of the ophthalmic system can be recognized: the lateral and medial branches (Figures 5(a) and 5(b)). The lateral branches include the lacrimal and recurrent meningeal branches which establish connections with external carotid branches, as the middle meningeal artery, along the dura over the greater sphenoid wing. The medial division includes the anterior and posterior ethmoidal branches that reach the anterior fossa and frontal pole after coursing through the ethmoidal canals. The continuation of the anterior ethmoidal artery along the falx is called falcine artery and is involved in the supply of the falx and frontal pole dura and in lesions involving these structures [1].

The main orbital drainage pathway is the superior ophthalmic vein (Figures 5(c) and 5(d)). This vein is formed by gathering of several tributaries on the medial orbital corner while the inferior ophthalmic vein is formed along the lateral orbital corner. These veins are connected along the base of the orbital pyramid by large anastomotic channels formed by the facial and angular veins. Posteriorly, these veins drain into the venous space of the cavernous sinus.

\section{Orbital Nerves}

Seven are also the orbital nerves (Figure 6). With the exception of the optic nerve which transits through the optic canal, all others pass along the superior orbital fissure. The 


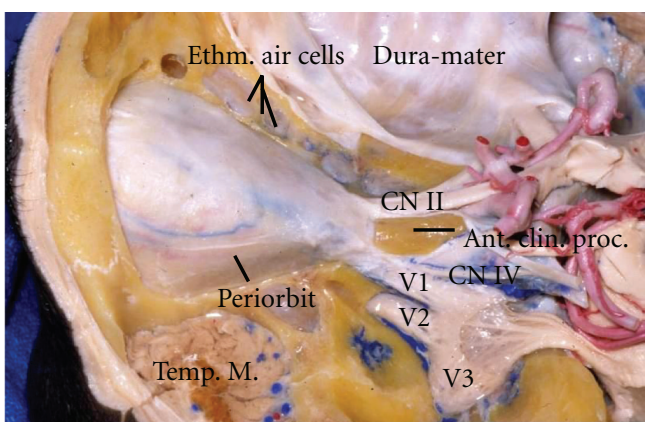

(a)
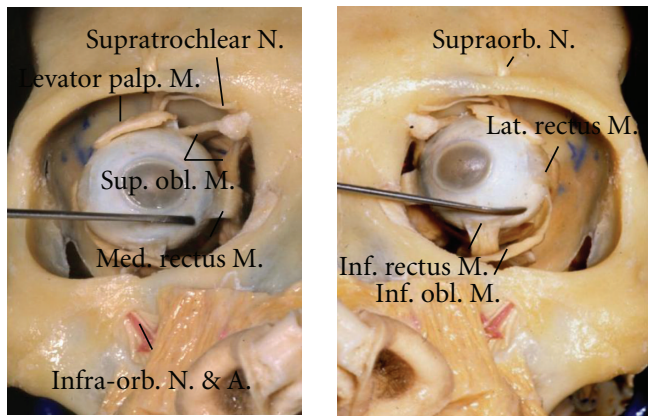

(b)

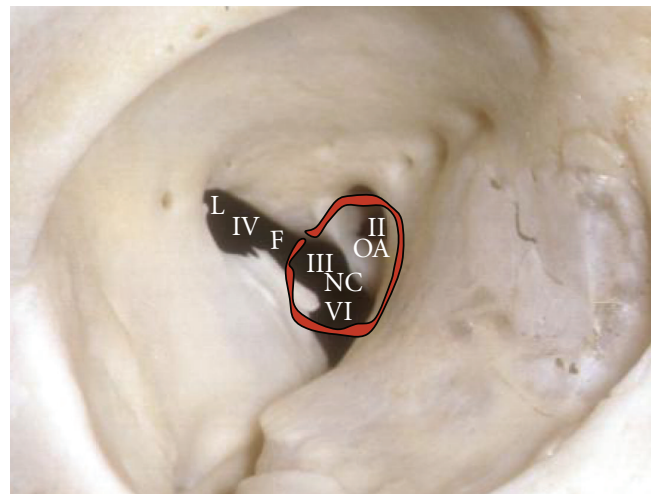

(c)

Figure 3: (a) The roof and lateral wall of the left orbit have been removed. Beyond the optic canal and superior orbital fissure, the bony orbit is covered by an extension of the cranial dura mater, the periorbit. The periorbit or orbital dura envelops and holds the intraorbital structures, especially the orbital fat. (b) With the exception of the orbicularis oculi muscle (Figure 1(a)), located along the base of the orbital pyramid, seven are the intraorbital muscles: levator palpebrae, superior, inferior, lateral, and medial rectus, and superior and inferior oblique muscles. Of these, only the inferior oblique muscle is attached to the medial orbital wall. The four recti and the superior oblique muscles attach along the orbital apex in and around a common annular tendon or fibrous ring, the annulus of Zinn. (c) The annulus inserts along the orbital apex, encircling the orbital opening of the optic canal and the central part of the superior orbital fissure. Structures coursing through the annulus are the optic nerve (II) and ophthalmic artery (OA), the oculomotor (III) and abducens (VI) nerves, and the nasociliary part $(\mathrm{N})$ of the ophthalmic division of the trigeminal nerve. The troclear nerve (IV) the frontal (F) and lacrimal (L) divisions of the trigeminal nerve course outside the annulus, on the lateral sector of the superior orbital fissure.

optic canal and the superior orbital fissure are separated by the optic strut, which is exposed by removing the anterior clinoid process (Figure 6(a)).

In the orbital apex, the trochlear nerve and the ophthalmic division of the trigeminal nerve are the most superficial structures (Figure 6(b)). The ophthalmic division gives off the lacrimal, frontal, and nasociliary nerves. The lacrimal nerve runs superficial to the annular tendon, above the superior ophthalmic vein, transmits the secretory fibers borrowed from the zygomatic nerve to the gland, and ends up conveying sensation from the area in front of the lacrimal gland. The frontal nerve gives further the supraorbital and supratrochlear divisions. The supratrochlear nerve runs anteriorly above the trochlea. The supraorbital nerve courses above the levator palpebrae muscle to convey sensation from the upper eyelid and forehead and may transmit autonomic fibers from the cervical sympathetic ganglia to the globe and pupillary dilator. The trochlear nerve, which also courses outside the annulus, reaches the superior oblique muscle, on the medial part of the orbit (Figure 6(c)).
The nasociliary nerve, the third part of the ophthalmic division, arises from its medial surface and separates into its nasal and ciliary portions along the orbital apex (Figure 6(d)).

The nasal part passes inside the annular tendon, above the optic nerve, directs to the medial orbital wall, and forms the anterior and posterior ethmoidal nerves (Figure 6(e)). The ciliary part also enters the orbit deep to the annulus, in the lateral space of the orbit, forms the sensory root, and passes the sympathetic fibers to the ciliary ganglion. Fibers from the sensory root pass to the globe through the short ciliary nerves and convey sensation to cornea and globe. The long ciliary nerves convey sympathetic fibers to the globe and pupillary dilator. The sympathetic fibers entering the ganglion emerge without synapsing in the ciliary ganglion.

It is important to understand the relationships of the ophthalmic division and the autonomic fibers. While the oculomotor nerve really conveys parasympathetic output (motor root) to the ciliary ganglion, several portions of the trigeminal division and abducens nerve transmit 


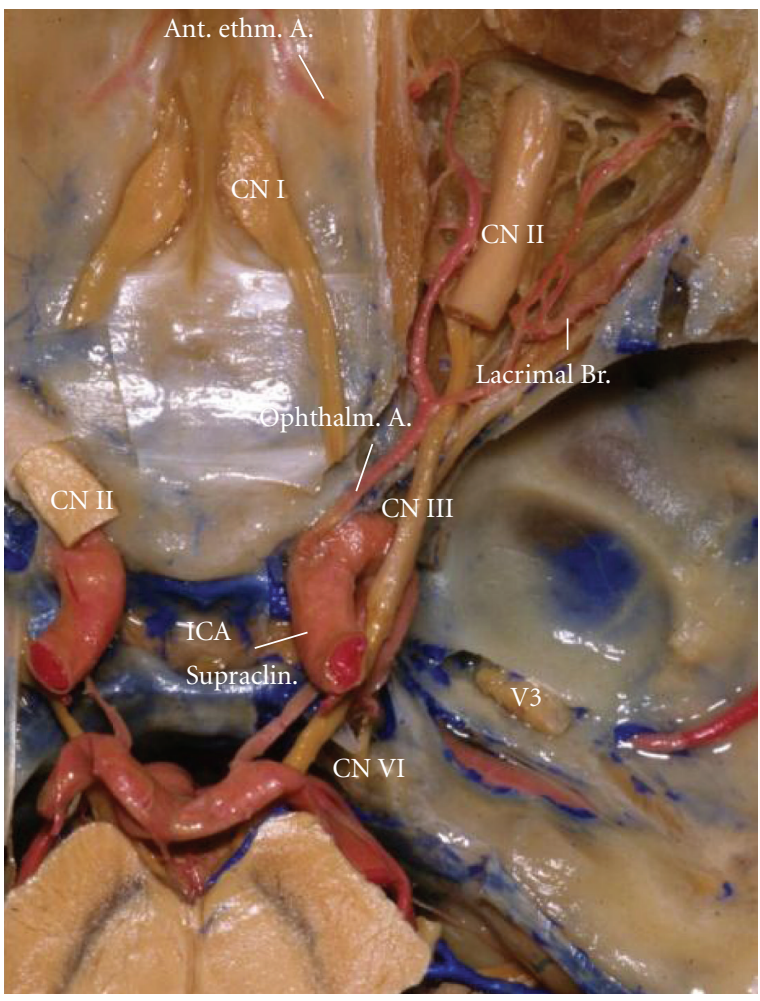

(a)

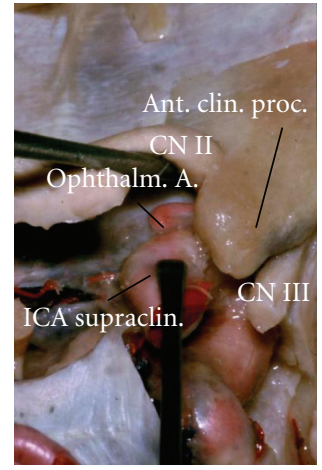

(b)

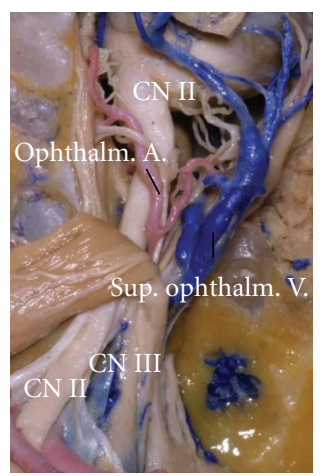

(d)

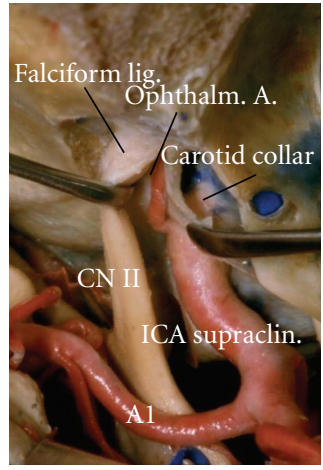

(c)

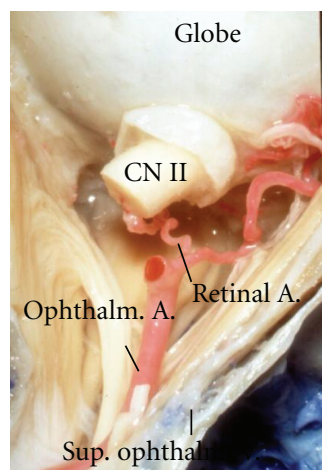

(e)

FIgure 4: (a)-(e) Dissections of the right cranial base and orbit. (a) The ophthalmic artery is a branch of the supraclinoid portion of the internal carotid artery, in most cases. This vessel follows the optic nerve in the optic canal and orbit and is the responsible for the supply of orbital structures. (b) The origin of the ophthalmic artery is usually medial to the anterior clinoid process, below the optic nerve. (c) At the optic canal, the artery has passed to a position lateral to nerve. This anatomical fact must be remembered during the opening of the falciform ligament after anterior clinoidectomy, to avoid iatrogenic lesion to this artery. (d) Once inside the orbit, the ophthalmic artery passes, in most cases, above the optic nerve. (e) At the point where the artery crosses the optic nerve, it gives rise to the central retinal artery. Working along the lateral orbital space, it is vital to avoid blind coagulation as ligation of the central retinal artery results in loss of vision.

sympathetic fibers originated from the cervical sympathetic chain that initially course around the cavernous carotid artery. This is the case with the frontal branch and the ciliary portion of the nasociliary nerve. The lacrimal nerve in its trajectory also serves as a "bridge" to sympathetic twigs directed to the lacrimal gland. These twigs are related to the pterygopalatine ganglion and initially transmitted through the zygomatic branch of the maxillary division, which course along the lateral orbital wall as the zygomatico-orbital nerves, to end as zygomaticofacial and zygomatico-orbital sensory branches along the cheek and temple. The ophthalmic artery also transmits sympathetic fibers.

Medial to the nasociliary nerve are the abducens and the oculomotor nerves (Figure 6(f)). The abducens is formed by the union of several twigs inside the cavernous sinus. It passes inside the annulus and, in the orbit, ends up spreading along the inner surface of the rectus lateralis.

At the orbital apex, the oculomotor nerve separates into superior and inferior divisions (Figures 6(g) and 6(h)). The superior division, courses deep to the annulus, directs above, and supplies the superior muscular complex, comprising the levator palpebrae and superior rectus muscle. The inferior division, coursing near the orbital floor, supplies the inferior and medial recti and the inferior oblique muscle. From the branch directed to the inferior oblique muscle an ascending twig reaches the ciliary ganglion, forming its parasympathetic root. The parasympathetic fibers synapse in the ganglion and continue as the short ciliary nerves to the pupillary sphincter.

\section{Topographical Relationships and Orbital Surgical Approaches}

The ethmoidal canals, the periorbit, the muscular cone, the optic nerve, and the superior orbital fissure are important structures in the topographical arrangement of the orbit.

The cranial openings of the ethmoidal canals are related with the anterior and posterior limits of the ethmoidal cribriform plate. Anterior to the anterior ethmoidal canal is the frontal portion of the anterior fossa, and posterior to the posterior canal is the area of the planum sphenoidale. This anatomical fact has been used as a topographical landmark during endonasal approaches to the anterior fossa. Similarly, the position of the orbital opening of the ethmoidal canals can also be useful to separate different orbital regions: 


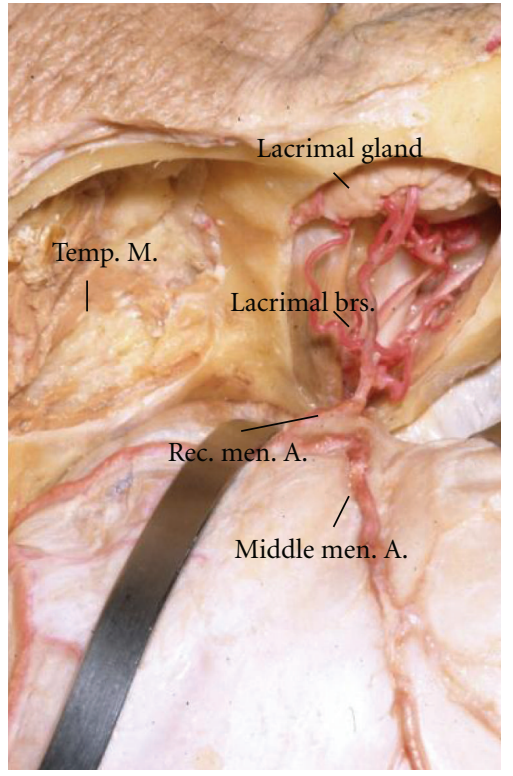

(a)

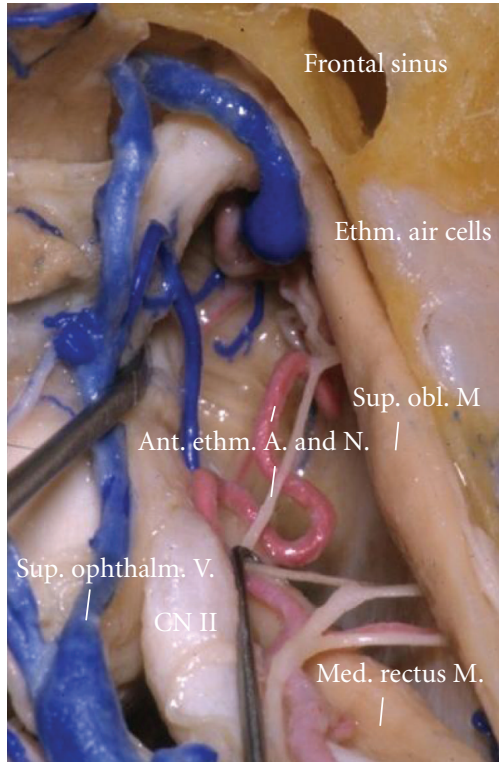

(c)

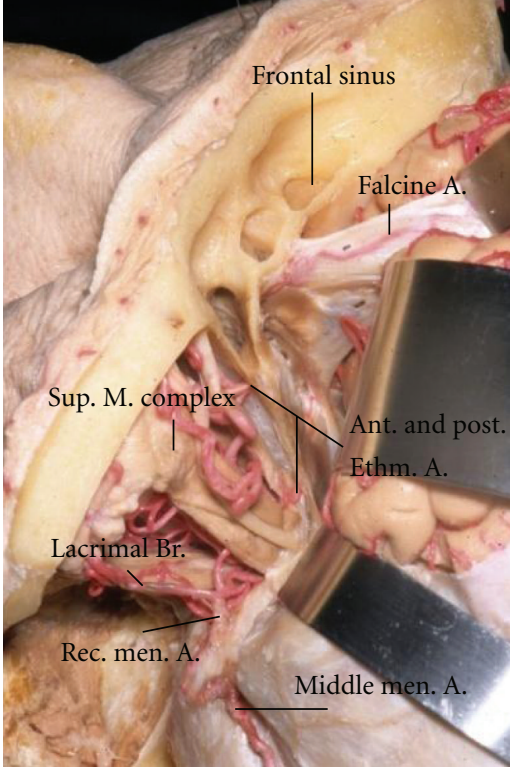

(b)

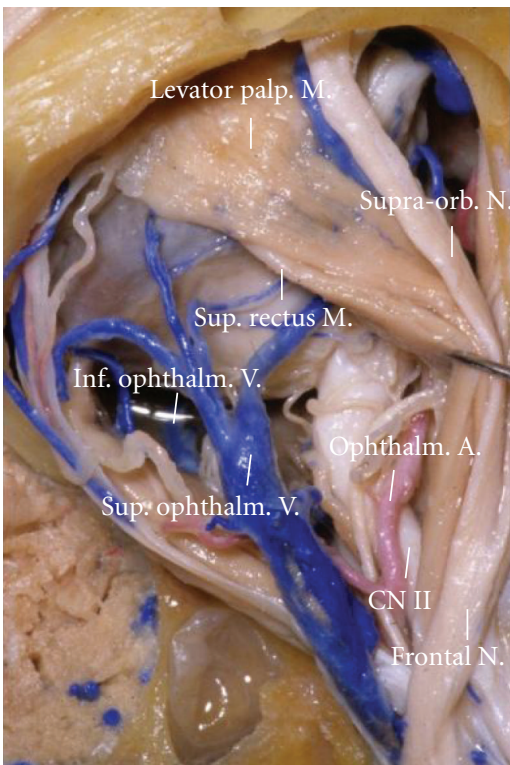

(d)

Figure 5: (a)-(d) Dissections of the left cranial base and orbit. The ophthalmic artery also sends dural branches and may thus become involved in the supply of dural based lesions. In this role, two sets of branches can be recognized: the lateral and medial branches of the ophthalmic system. (a) The lateral branches include the lacrimal, recurrent meningeal, and sphenoidal branches which establish connections with external carotid branches (middle and accessory meningeal artery) along the dura over the greater sphenoid wing. (b) The medial division includes the anterior and posterior ethmoidal branches that reach the anterior fossa and frontal pole after coursing through the ethmoidal canals. The continuation of the anterior ethmoidal artery along the falx is named falcine artery and is involved in the supply of the falx and dura over the frontal pole. (c) The main orbital drainage pathway is the superior ophthalmic vein. This vein forms by gathering of several tributaries on the medial orbital corner. (d) From the medial orbital corner, the superior ophthalmic vein crosses above the optic nerve, collecting, on occasions, the smaller inferior ophthalmic vein. In this specimen, the superior ophthalmic vein passes outside the annulus to end up into the cavernous sinus.

anterior to the anterior ethmoidal canal is the bulbar part of the orbit. Between both canals is the retrobulbar part, and posterior to the posterior ethmoidal canals is the orbital apex (Figure $7(\mathrm{a})$ ). The seven also rules here: the anterior ethmoidal canal is on average $21 \mathrm{~mm}$ from the medial orbital edge. The posterior ethmoidal canal is around $14 \mathrm{~mm}$ posterior to the anterior canal, and the optic canal is on average $7 \mathrm{~mm}$ posterior to the posterior canal (Figure 7(b)).

The superior orbital fissure separates the orbit, anteriorly, from the cavernous sinus, posteriorly [5]. Orbital lesions 


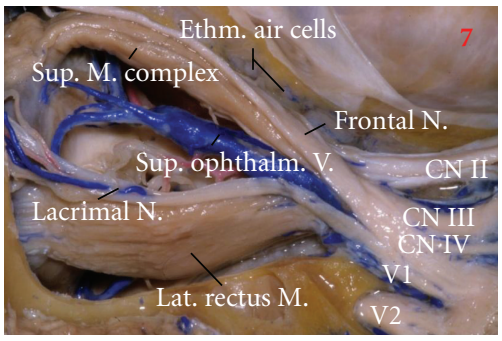

(a)

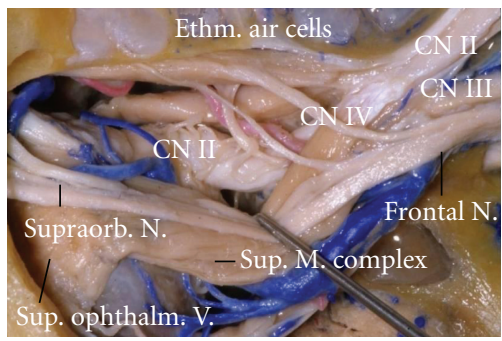

(c)

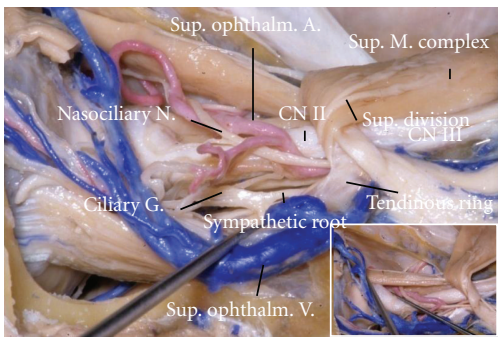

(e)

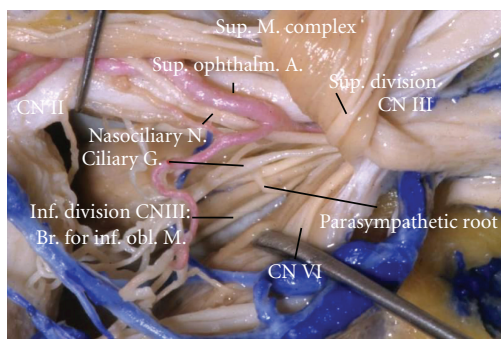

(g)

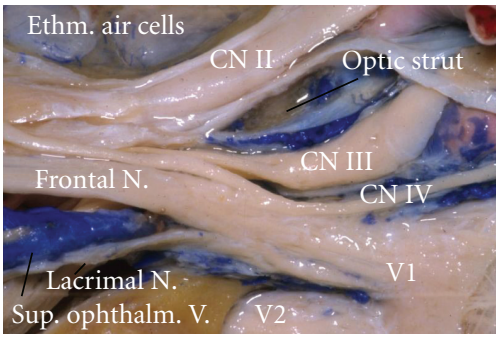

(b)

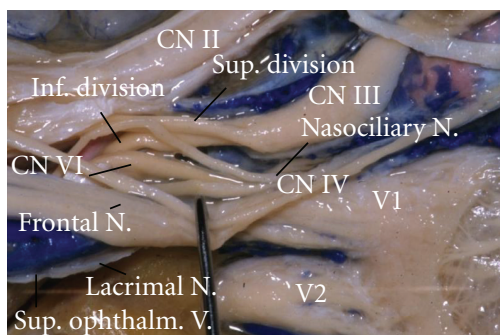

(d)

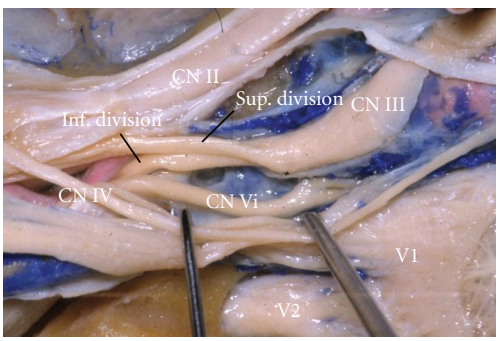

(f)

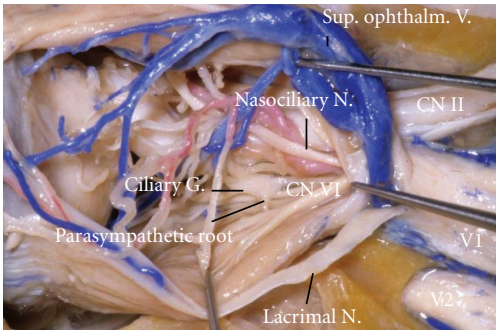

(h)

Figure 6: (a) Dissection of the left orbit has proceeded from the point seen on Figure 3(a). The periorbit and orbital fat have been dissected and the anterior clinoid removed, to expose the orbital apex. (b) The optic strut separates the optic canal and the superior orbital fissure. Seven are the orbital nerves: optic, oculomotor, trochlear, frontal, lacrimal, nasociliary, and abducens nerves. With the exception of the optic nerve which transits through the optic canal, all others pass along the superior orbital fissure. In the orbital apex, the trochlear nerve and the ophthalmic division of the trigeminal nerve are the most superficial structures. The ophthalmic division gives off the lacrimal nerve, which courses towards the lacrimal gland and the frontal nerve. (c) The frontal nerve gives further the supraorbital and supratroclear divisions. The troclear nerve, which also courses outside the annulus, reaches the superior oblique muscle, on the medial part of the orbit. (d) The nasociliary nerve, the third part of the ophthalmic division, arises from its medial surface and separates into nasal and ciliary portions along the orbital apex. (e) The nasal part passes inside the annular tendon, above the optic nerve, and directs to the medial orbital wall to forms the anterior and posterior ethmoidal nerves (Insert). The ciliary part also enters the orbit deep to the annulus and, in the lateral space of the orbit, forms the sympathetic root of the ciliary ganglion and the long ciliary nerves. The sympathetic fibers emerge without synapsing in the ciliary ganglion. (f)-(h) The abducens and oculomotor nerves are found medially. The abducens is formed by the union of several twigs inside the cavernous sinus. It passes inside the annulus and, in the orbit, ends up spreading along the inner surface of the rectus lateralis muscle (g). At the orbital apex, the oculomotor nerve separates into superior and inferior divisions. The superior division courses deep to the annulus, directs above, and supplies the superior muscular complex, comprising the levator palpebrae and superior rectus muscle (g). The inferior division, coursing near the orbital floor, supplies the inferior and medial recti and the inferior oblique muscle. From the branch directed to the inferior oblique muscle, an ascending twig reaches the ciliary ganglion ( $\mathrm{g}$ and $\mathrm{h}$ ), forming its parasympathetic root. The parasympathetic fibers synapse in the ganglion and continue as the short ciliary nerves to the pupillary sphincter. 


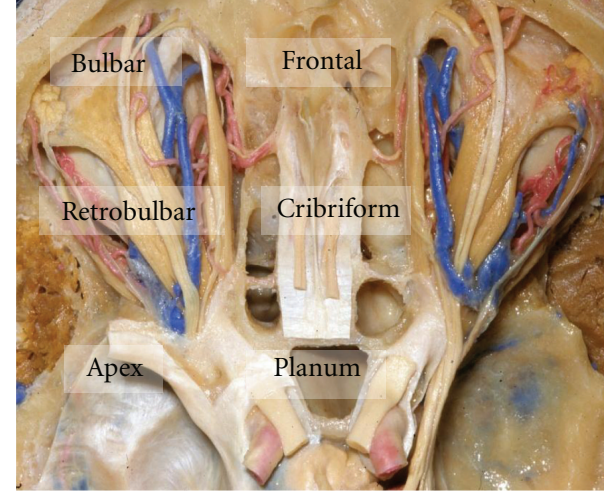

(a)

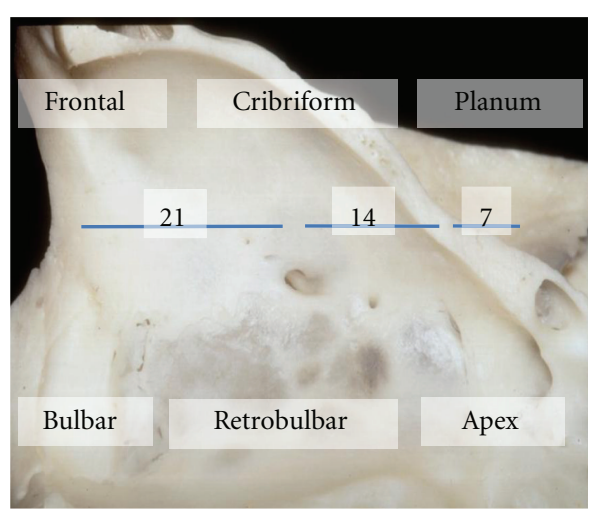

(b)

FIGURE 7: (a) The anterior fossa floor and roof of the orbit have been removed and the orbital contents dissected to expose the topographical relationships between the ethmoidal canals and the roof of the nasal cavity and orbit. The ethmoidal canals are related with the anterior and posterior limits of the ethmoidal cribriform plate. Anterior to the anterior ethmoidal canal is the frontal portion of the anterior fossa, and posterior to the posterior canal is the area of the planum sphenoidale. Similarly, anterior to the anterior ethmoidal canal is the bulbar part of the orbit, between both canals is the retrobulbar part, and posterior to the posterior ethmoidal canals is the orbital apex. (b) Sagittal cut through the left orbit, exposing its medial wall. The anterior ethmoidal canal is on average $21 \mathrm{~mm}$ from the medial orbital edge. The posterior ethmoidal canal is around $14 \mathrm{~mm}$ posterior to the anterior canal, and the optic canal is on average $7 \mathrm{~mm}$ posterior to the posterior canal.

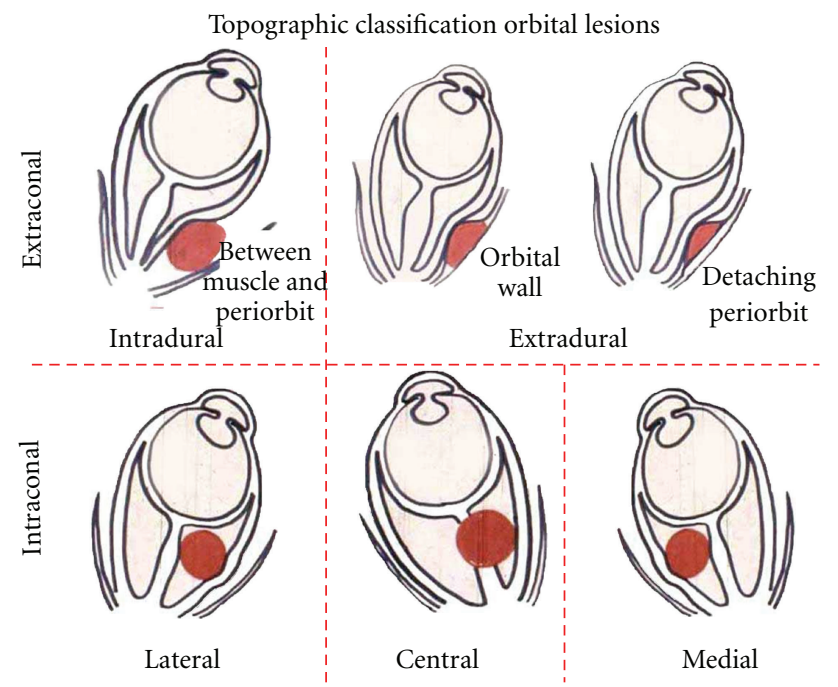

Figure 8: Topographic classification of orbital lesions. The periorbit, the muscular cone, the optic nerve, and the superior orbital fissure are important structures in the topographical arrangement of the orbit. The presence of the periorbit allows classifying the orbital lesions in intradural — when deep to the periorbit—and extradural—when located between the periorbit and the bony orbit. The muscular cone divides the orbit into intraconal and extraconal spaces. While the intraconal lesions are always intradural, the extraconal lesion can be either intra- or extradural. The intraconal space also can be further subdivided in relation to the optic nerve into medial, central, and lateral orbital spaces.

that extend beyond the fissure, towards the cavernous sinus, might require combined surgical approaches to these areas, doubtless including orbital lesions into the realm of skull base surgery.

The presence of the periorbit allows classifying the orbital lesions in intradural-when deep to the periorbit-and extradural-when located between the periorbit and the bony orbit (Figure 8 ). The muscular cone divides the orbit into intraconal and extraconal spaces. While the intraconal lesions are always intradural, the extraconal lesion can be either intra- or extradural. The intraconal space also can be further subdivided in relation to the optic nerve into medial, central, and lateral orbital spaces.

This understanding is paramount in classifying the orbital lesions and in adequately choosing a surgical path for its treatment.

The earliest reports of surgery for orbital lesions involved approaches directed through the lateral wall of the orbit $[6,7]$. The first report of a transcranial approach to the orbit was published in 1922 by Dandy [8]. Since then, both 


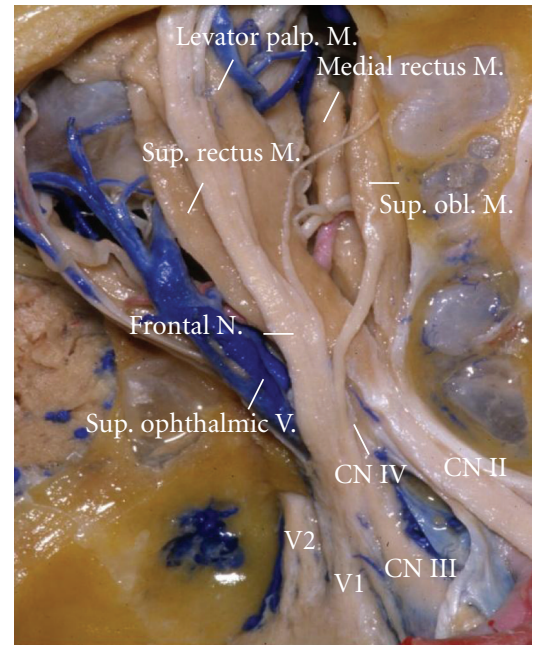

(a)

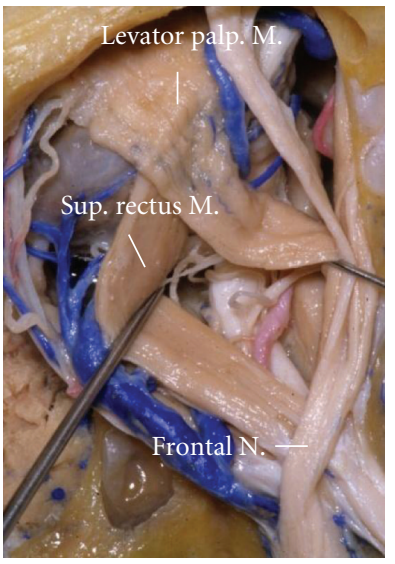

(c)

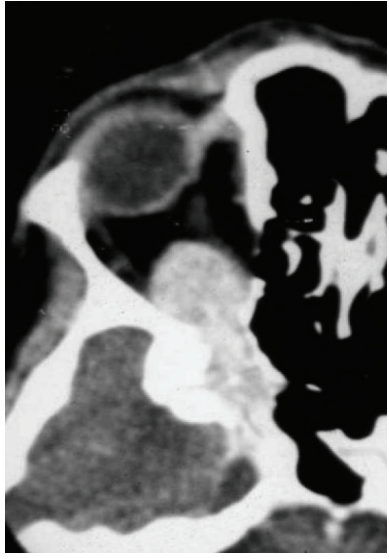

(d)

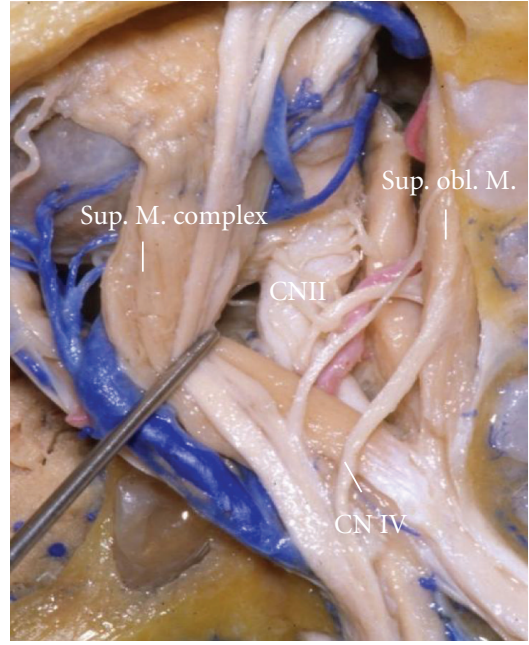

(b)

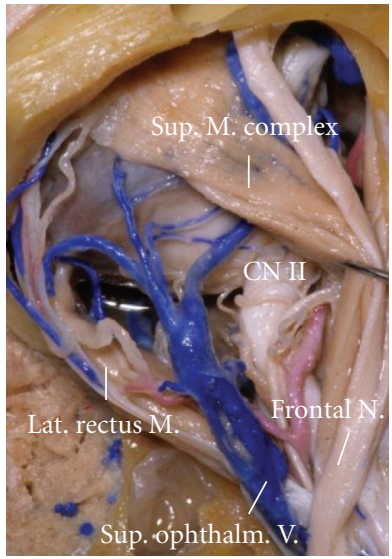

(e)

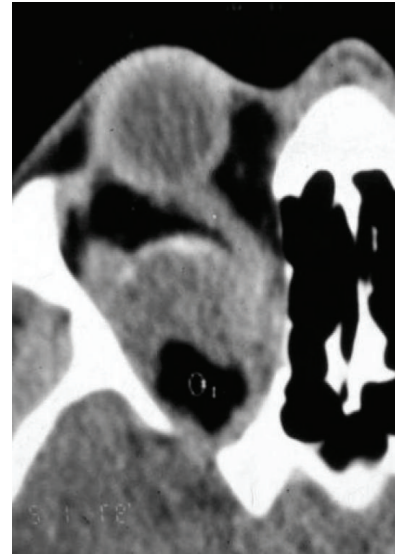

(f)

FIGURE 9: (a) Intraorbital routes. The left orbit is seen from above as in a cranio-orbital approach. Medial, central, and lateral routes can be chosen pending on the location of the lesion to the optic nerve. (b) The medial route is performed between the superior oblique and superior muscular complex. This route gives access to the optic nerve from the globe to apex. The annulus can be opened for further access to the apex, with careful handling of the troclear nerve which courses from lateral to medial in this area. The medial space can also be reached by working endoscopically through the nasal cavity and medial orbital wall, beyond the medial rectus muscle. (c)-(d) The central route is taken by opening the periorbit and splitting the superior muscular complex. This is the shortest route to the central part of the optic nerve and to lesion situated along this area as a meningioma of the optic nerve sheath (d). The central route has two variants, pending on traction over the frontal nerve, which can be retracted medially, with the levator palpebrae muscle or laterally with the superior. On this last variant, access granted to the apical part of the optic nerve is broader, but it requires not only dissecting and displacing the frontal nerve but also opening the orbital septum. (e)-(f) The lateral intraorbital route gives access to the major space around the optic nerve. This space can be reached from above, by working between the superior muscular complex and lateral rectus muscle or laterally after a lateral orbitotomy is accomplished and is suitable for lesions located in this area, as orbital hemangiomas (f).

extra- and intracranial routes to orbital lesions have been developed [9-11]. The transcranial approach is commonly selected for tumors located in the orbital apex and/or optic canal, or involving both the orbit and adjacent intracranial areas $[8,12,13]$. Tumors confined within the periorbit in the anterior two thirds of the orbit can often be approached extracranially, but those located in the apical area, and especially those on the medial side of the optic nerve, often require a transcranial approach. An approach directed through the lateral orbital wall, involving an osteotomy of lateral rim and wall, is commonly selected for tumors confined to the superior, lateral, or inferior compartment of the orbit and those in the lateral part of the apex $[6,7]$. An approach directed along the medial orbital wall may be used for tumors located medial to the optic nerve that are not located deep in the apex $[7,14,15]$. The transcranial surgical approaches to the orbit may be arbitrarily divided into two types based on whether the orbital rim is or is not elevated in exposing the orbital lesion. Early approaches involved removal of a frontal or frontotemporal bone flap, with preservation of the supraorbital rim, and opening of the orbit behind the rim [10, 16-22].

An approach directed through the lateral orbital wall, involving an osteotomy of the lateral orbital rim and wall, 
is selected for tumors confined to the superior, temporal, or inferior compartment of the orbit and those in the lateral part of the apex $[6,7]$.

The transmaxillary approach, directed through the orbital floor, is most commonly performed using a sublabial incision in the gingivobuccal margin [23, 24], or more recently with endoscopic assistance.

The medial orbital incision can be used to provide access to the area lateral to the lacrimal and ethmoid bones back to the orbital apex, and with removal of some of the ethmoid air cells and sphenoid sinus facing the orbit, the optic canal can be exposed or decompressed [23, 24].

As it happens in the skull, where a specific approach might give rise to several possible intracranial routes, a similar reasoning is possible in the orbit. The intraorbital routes obey to the spaces related to the optic nerve and can be divided into central, lateral, or medial to the nerve (Figure 9) $[3,5,25,26]$.

The medial route is performed between the superior oblique and superior muscular complex (Figures 9(a) and 9(b)). This route gives access to the optic nerve from the globe to apex. The annulus can be opened for further access to the apex, with careful handling of the trochlear nerve which courses from lateral to medial in this area. The medial space can also be reached by working endoscopically through the nasal cavity and medial orbital wall, beyond the medial rectus muscle.

The central route is achieved by opening the periorbit and splitting the superior muscular complex. This is the shortest route to the central part of the optic nerve (Figures $9(\mathrm{c})$ and $9(\mathrm{~d}))$. The central route has two variants, pending on traction over the frontal nerve, which can be retracted medially, with the levator palpebrae muscle or laterally with the superior rectus. On this last variant, access granted to the apical part of the optic nerve is broader, but it requires not only dissecting and displacing the frontal nerve but also opening the orbital septum.

The lateral intraorbital route gives access to the major space around the optic nerve (Figures $9(\mathrm{e})$ and $9(\mathrm{f})$ ). This space can be reached from above, by working between the superior muscular complex and lateral rectus muscle, or laterally after a lateral orbitotomy is accomplished.

\section{Conclusion}

Knowledge of orbital osteology is paramount in adequately choosing and performing an orbital approach. Understanding the critical topographical elements in this area helps to classify an orbital lesion and provides for a solid basis in choosing the most adequate intraorbital route for its treatment.

\section{References}

[1] C. Martins, A. Yasuda, A. Campero, A. J. Ulm, N. Tanriover, and A. L. Rhoton Jr., "Microsurgical anatomy of the dural arteries," Operative Neurosurgery, vol. 56, no. 2, pp. 1-41, 2005.

[2] F. S. Harris and A. L. Rhoton Jr., "Anatomy of the cavernous sinus. A microsurgical study," Journal of Neurosurgery, vol. 45, no. 2, pp. 169-180, 1976.
[3] A. L. Rhoton Jr. and Y. Natori, The Orbit and Sellar Region: Microsurgical Anatomy and Operative Approaches, Thieme Medical, New York, NY, USA, 1966.

[4] Q. Liu and A. L. Rhoton Jr., "Middle meningeal origin of the ophthalmic artery," Neurosurgery, vol. 49, no. 2, pp. 401-407, 2001.

[5] Y. Natori and A. L. Rhoton Jr., "Microsurgical anatomy of the superior orbital fissure," Neurosurgery, vol. 36, no. 4, pp. 762775, 1995.

[6] RU Krönlein, “Zur Pathologie und operativen Behandlung der Dermoidcysten der Orbita," Beitrag Klinische Chirurgie, vol. 4, pp. 149-163, 1889.

[7] J. C. Maroon and J. S. Kennerdell, "Surgical approaches to the orbit. Indications and techniques," Journal of Neurosurgery, vol. 60, no. 6, pp. 1226-1235, 1984.

[8] W. E. Dandy, "Prechiasmal intracranial tumors of the optic nerves," American Journal of Ophthalmology, vol. 5, no. 3, pp. 169-188, 1922.

[9] O. Al-Mefty and J. L. Fox, "Superolateral orbital exposure and reconstruction," Surgical Neurology, vol. 23, no. 6, pp. 609$613,1985$.

[10] E. M. Housepian, "Surgical treatment of unilateral optic nerve gliomas," Journal of Neurosurgery, vol. 31, no. 6, pp. 604-607, 1969.

[11] J. A. Jane, T. S. Park, L. H. Pobereskin, H. R. Winn, and A. B. Butler, "The supraorbital approach: technical note," Neurosurgery, vol. 11, no. 4, pp. 537-542, 1982.

[12] W. Hassler and H. R. Eggert, "Extradural and intradural microsurgical approaches to lesions of the optic canal and the superior orbital fissure," Acta Neurochirurgica, vol. 74, no. 3-4, pp. 87-93, 1985.

[13] E. M. Housepian, "Microsurgical anatomy of the orbital apex and principles of transcranial orbital exploration," Clinical Neurosurgery, vol. 25, pp. 556-573, 1978.

[14] S. E. Kelman, R. Heaps, A. Wolf, and M. J. Elman, "Optic nerve decompression surgery improves visual function in patients with pseudotumor cerebri," Neurosurgery, vol. 30, no. 3, pp. 391-395, 1992.

[15] S. Niho, M. Niho, and K. Niho, "Decompression of the optic canal by the transethmoidal route and decompression of the superior orbital fissure," Canadian Journal of Ophthalmology, vol. 5, no. 1, pp. 22-40, 1970.

[16] C. H. Frazier, "An approach to the hypophysis through the anterior cranial fossa," Annals of Surgery, vol. 57, pp. 145-150, 1913.

[17] W. B. Hamby, "Pterional approach to the orbits for decompression or tumor removal," Journal of Neurosurgery, vol. 21, pp. 15-18, 1964.

[18] H. Jackson, "Orbital tumours," Proceedings of the Royal Society of Medicine, vol. 38, pp. 587-594, 1945.

[19] J. G. Love and W. L. Benedict, "Transcranial removal of intraorbital tumors," Journal Of the American Medical Association, vol. 129, pp. 777-784, 1945.

[20] C. S. MacCarty and D. N. Brown, "Orbital tumors in children," Clinical Neurosurgery, vol. 11, pp. 76-93, 1964.

[21] L. L. McArthur, "An aseptic surgical access to the pituitary body and its neighborhood," Journal Of the American Medical Association, vol. 58, pp. 2009-2011, 1912.

[22] H. C. Naffziger, "Progressive exophthalmos following thyroidectomy: its pathology and treatment," Annals of Surgery, vol. 94, pp. 582-586, 1931.

[23] T. Hitotsumatsu and A. L. Rhoton Jr., "Unilateral upper and lower subtotal maxillectomy approaches to the cranial 
base: microsurgical anatomy," Neurosurgery, vol. 46, no. 6, pp. 1416-1453, 2000.

[24] T. Hitotsumatsu, T. Matsushima, and A. L. Rhoton Jr., "Surgical anatomy of the midface and the midline skull base," in Operative Techniques in Neurosurgery, R. F. Spetzler, Ed., vol. 2, pp. 160-180, W. B. Saunders, 1999.

[25] Y. Natori and A. L. Rhoton Jr., "Transcranial approach to the orbit: microsurgical anatomy," Journal of Neurosurgery, vol. 81, no. 1, pp. 78-86, 1994.

[26] A. L. Rhoton Jr., “The orbit," Neurosurgery, vol. 51, no. 4, supplement 1, pp. 303-334, 2002. 

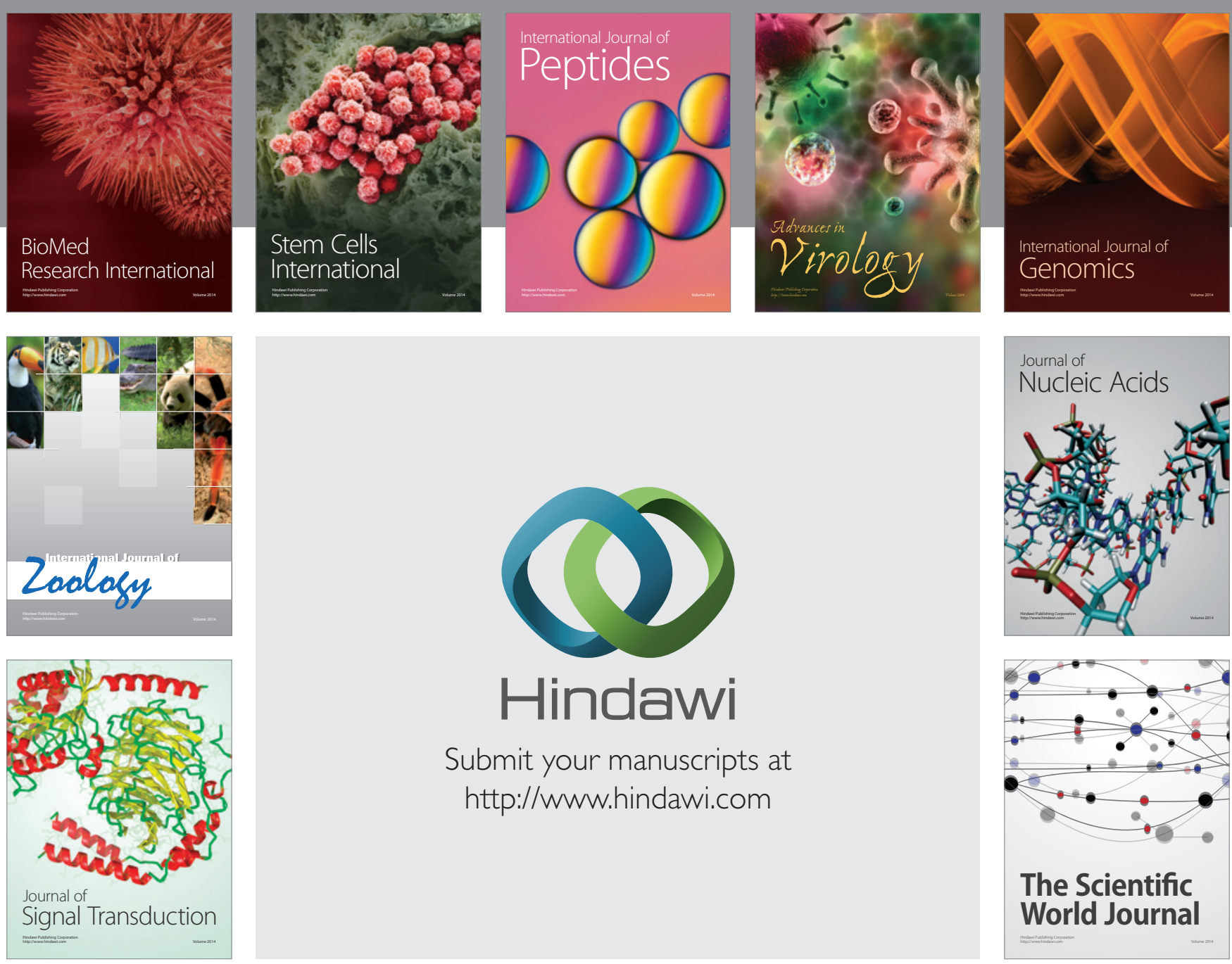

Submit your manuscripts at

http://www.hindawi.com
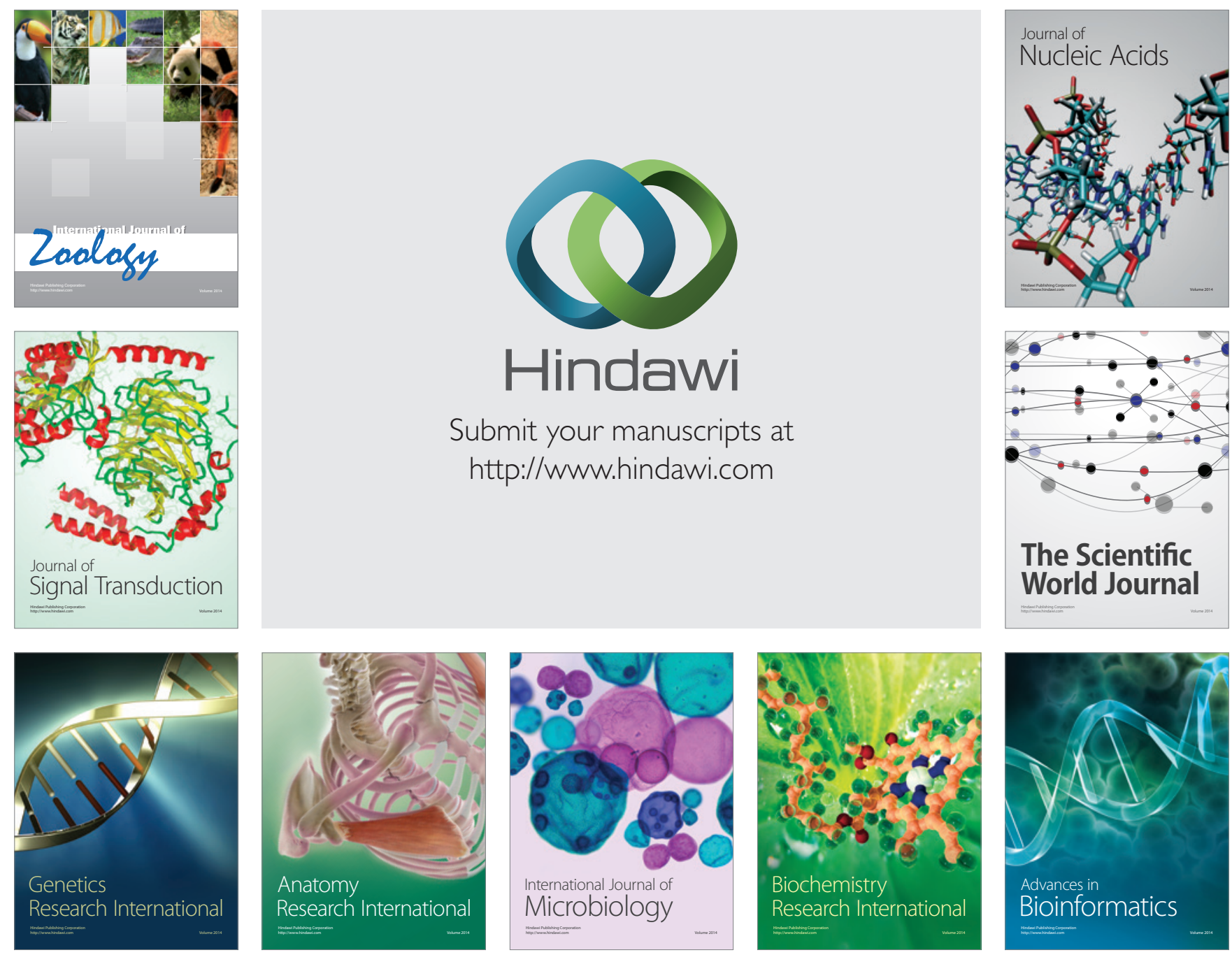

The Scientific World Journal
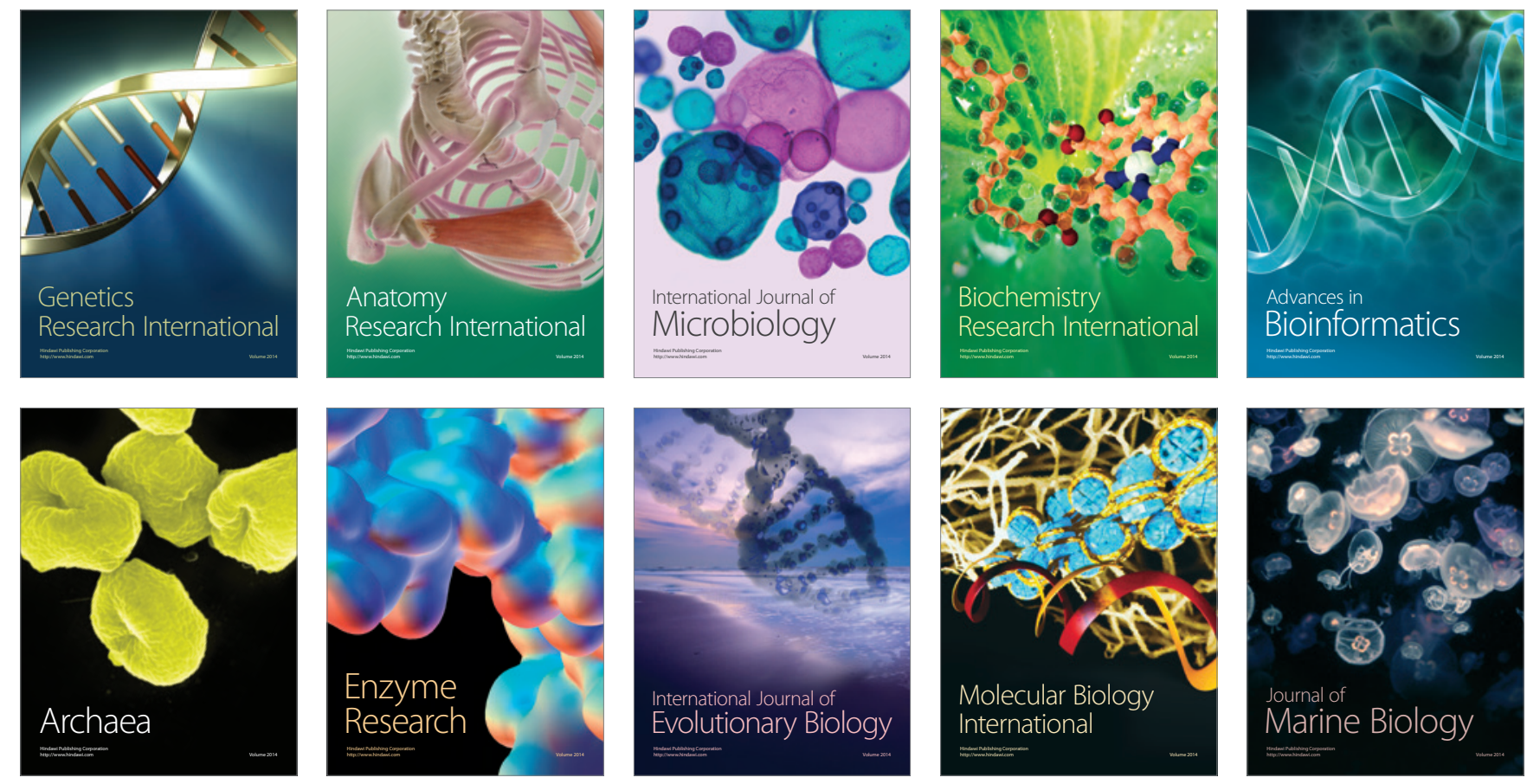